\title{
p21 loss compromises the relative quiescence of forebrain stem cell proliferation leading to exhaustion of their proliferation capacity
}

\author{
Tod E. Kippin, ${ }^{1}$ David J. Martens, and Derek van der Kooy \\ Neurobiology Research Group, Department of Medical Genetics and Microbiology, University of Toronto, \\ Toronto, Ontario M5S 1A8, Canada
}

\begin{abstract}
Adult stem cells in various tissues are relatively quiescent. The cell cycle inhibitor $\mathbf{p}^{21^{\text {cip } 1 / w a f 1}}$ (p21) has been shown to be important for maintaining hematopoietic stem cell quiescence and self-renewal. We examined the role of $\mathbf{p} 21$ in the regulation of adult mammalian forebrain neural stem cells (NSCs). We found that $p 21^{-/-}$ mice between post-natal age 60-240 d have more NSCs than wild-type $(+/+)$ controls due to higher proliferation rates of $p 21^{-/-}$NSCs. Thereafter, NSCs in $p 21^{-/-}$mice decline and are reduced in number at 16 mo relative to $p 21^{+/+}$mice. Similarly, both $p 21^{-/-}$and $p 21^{+/+}$NSCs display self-renewal in vitro; however, $p 21^{-/-}$NSCs display limited in vitro self-renewal (surviving a few passages, then exhausting). Thus, p21 contributes to adult NSC relative quiescence, which we propose is necessary for the life-long maintenance of NSC self-renewal because NSCs may be limited to a finite number of divisions.
\end{abstract}

[Keywords: Aging; cell cycle; neural stem cell; neurogenesis; $\left.221^{\text {cip1/waf1 }}\right]$

Supplemental material is available at http://www.genesdev.org.

Received October 15, 2004; revised version accepted February 3, 2005.

The adult mammalian brain contains neural stem cells (NSCs) that have the ability to proliferate and generate multipotential progeny throughout the lifetime of an organism (Gage 2000; van der Kooy and Weiss 2000; Seaberg and van der Kooy 2003). However, it is unclear whether NSCs have an unlimited capacity to proliferate (i.e., an infinite number of cell divisions). Stem cells have been described as having extensive proliferative capacity. In fact, NSC cultures can be maintained in vitro indefinitely (Reynolds and Weiss 1996), although this may be the result of cellular transformations after long periods in vitro (Morshead et al. 2002). Liver stem cells appear to have an unlimited ability to repopulate liver tissue during serial transplantation following liver damage (Overturf et al. 1997; Presnell et al. 2002). Similarly, transplanted hematopoietic stem cells can serially repopulate the entire blood lineage in irradiated recipients (for review, see Weissman 2000, 2001). However, the repopulation capacity of $p 21^{-/-}$hematopoietic stem cells is reduced following prior expansion (Cheng et al. 2000), suggesting that repopulation capacities are limited after many stem cell divisions. Consistent with this notion,

${ }^{1}$ Corresponding author.

E-MAIL kippin@psych.ucsb.edu; FAX (843) 792-4423.

Article and publication are at http://www.genesdev.org/cgi/doi/10.1101/ gad. 1272305 all nontransformed cells are believed to be capable of undergoing only a finite number of mitotic divisions (Hayflick 1965; Shay and Wright 2000). Furthermore, compared to transient progenitor cells, adult stem cells are relatively quiescent (i.e., low proliferation rate) in both blood (Mauch et al. 1995; Gardner et al. 1997) and brain (Morshead et al. 1994) and perhaps other tissues. It has been proposed that relative quiescence ensures stem cell longevity (i.e., long-term maintenance of self-renewal ability) by decreasing proliferation to prevent exhausting the limits of proliferation capacity (Ogawa 1994; Weiss and van der Kooy 1998). However, no studies have examined the mechanisms that regulate the extent of proliferative capacity of neural stem cells during aging. Here, we show that an initial increase in NSC expansion results in a decrement in long-term maintenance of NSCs, demonstrating that NSCs have a finite proliferation capacity.

In the present study, we show that there is an initial in vivo expansion of adult NSCs due to a reduction in relative quiescence following loss of the cyclin-dependent kinase inhibitor (CDKI), p2 $1^{\text {cip1/waf1 }}$ (p21 hereafter), followed by a diminished NSC maintenance capacity both in vivo and in vitro. Progression through the cell cycle is determined by the levels of cyclins and cyclin-dependent kinases (CDKs) that are negatively regulated by CDKIs (Musunuru and Hinds 1997). Loss-of-function studies re- 
veal that NSC proliferation is negatively regulated by the CDKI p27 ${ }^{\text {Kip } 1 ~(D o e t s c h ~ e t ~ a l . ~ 2002) ~ a n d ~ i t s ~ u p s t r e a m ~}$ regulator, Pten (Groszer et al. 2001). Conversely, p16Inka derepression in Bmi-1-deficient mice reduced NSC self-renewal, leading to NSC deletion during juvenile development (Molofsky et al. 2003). In addition, hematopoietic stem cell proliferation is also negatively regulated by p21 (Cheng et al. 2000). However, the effect of loss of CDKIs on in vivo adult NSC longevity has not been examined. We chose to investigate NSC longevity following the loss of $\mathrm{p} 21$ because $p 21^{-/-}$mice survive into late adulthood with a low incidence of tumorigenesis. Our findings demonstrate that p21 negatively regulates adult NSC proliferation and that NSCs have a finite proliferation potential.

\section{Results}

Loss of p21 increases expansion but reduces longevity of forebrain subependymal neural stem cells in vivo

The effect of $\mathrm{p} 21$ loss on the in vivo generation and maintenance of neurosphere-forming cells under clonal culture conditions (Reynolds and Weiss 1996; Tropepe et al. 1999; Morshead et al. 2003) was assessed in $p 21^{-/-}$ mice and their wild-type, B6Sf129, control strain $\left(p 21^{+/+}\right)$ from embryonic day 14 (E14) to post-natal $480 \mathrm{~d}$ of age. Loss of p21 did not alter the number of neurospheres derived from gross dissection of the ganglionic eminence at either E14 or post-natal 1-d-old mice (Fig. 1A). However, p21 plays a significant role in the regulation of neurosphere-forming cells in the adult brain, as revealed by a significant increase in the number of neurospheres derived from the subependyma of the rostral lateral ventricle at post-natal $60 \mathrm{~d}$ in $p 21^{-/-}$mice relative to $p 21^{+/+}$; this increase lasts until $\sim 240 \mathrm{~d}$ (Fig. 1B). Consistent with previous reports (Enwere et al. 2004; Kippin et al. 2004; Maslov et al. 2004), the numbers of neurospheres derived from the subependyma decline during aging in both $p 21^{+/+}$and $p 21^{-/-}$mice. Indeed, between 240 and $480 \mathrm{~d}$,

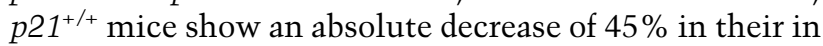
vivo neurosphere-forming cell population. Moreover, the rate of decline was accelerated in the $p 21^{-/-}$mice with an absolute decrease in neurosphere-forming cells of $>80 \%$ over the same period. Thus, significantly fewer neuro spheres were derived from $p 21^{-/-}$compared to $p 21^{+/+}$mice at $480 \mathrm{~d}$ of age (Fig. 1B). These data suggest that $p 21^{-/-}$ NSCs expand their population during early adult ages, but these NSCs are lost at a more rapid rate during aging.

Long-term (30 d) retention of bromo-deoxy-uridine (BrdU) labeling of NSCs in the subependyma in vivo confirmed the changes in NSC numbers resulting from $\mathrm{p} 21$ loss revealed by the neurosphere assay in vitro. Longterm retention of BrdU quantifies the proliferating portion of the relatively quiescent NSCs, but not the rapidly dividing progenitor cells that migrate out of the subependyma, undergo cell death, or dilute out the marker within $30 \mathrm{~d}$ (Morshead and van der Kooy 1992; Luskin 1993; Lois and Alvarez-Buylla 1994; Morshead et al. 1998; Hitoshi et al. 2002; Maslov et al. 2004). At $60 \mathrm{~d}$ of
A

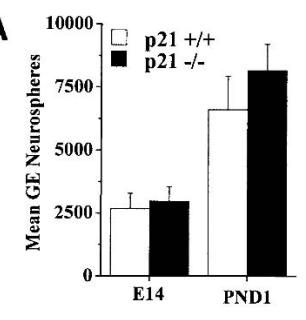

B
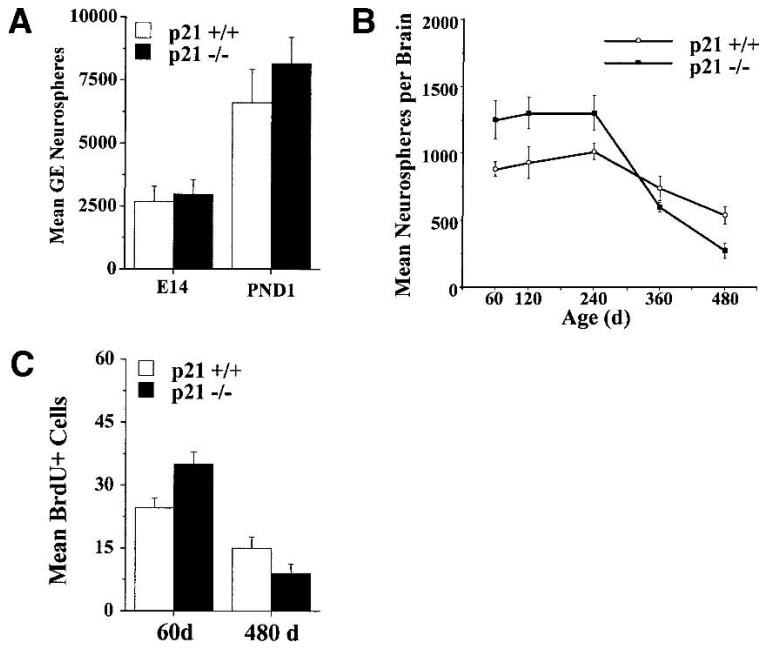

Figure 1. p21 loss results in an initial expansion, but decreased longevity, of neural stem cells in vivo. (A) Numbers (mean \pm SEM) of clonal neurospheres derived from the germinal eminence from $p 21^{+/+}$and $p 21^{-/-}$mice at E14 and $1 \mathrm{~d}$ of age. No differences between genotypes were observed. $(B)$ Numbers (mean \pm SEM) of clonal neurospheres derived from the rostral subependyma of the lateral ventricle from adult $p 21^{+/+}$and $p 21^{-/-}$mice at $60-480 \mathrm{~d}$ of age $(n=5-10$ per group per age). There was a significant interaction between genotype and age [F $(7,79)=4.44, p<0.05]$. Relative to $p 21^{+/+}$, the numbers of subependyma neurospheres from $p 21^{-/-}$mice were initially increased in young adults from 60 to $240 \mathrm{~d}$ of age ( $p s<0.05$ ) then decreased in old adults at $480 \mathrm{~d}$ of age $(p<0.05)$. (C) Numbers of long-term (30 d) BrdU-retaining cells in the rostral subependyma of the lateral ventricle in $p 21^{+/+}$and $p 21^{-/-}$mice at 60 and $480 \mathrm{~d}$ of age $(n=5-8$ per group per age); data are presented as mean \pm SEM per section of lateral ventricle subependyma. There was a significant interaction between genotype and age $[\mathrm{F}$ $(1,14)=9.61, p<0.05]$. The numbers of BrdU-labeled cells were increased at $60 \mathrm{~d}$ of age $(p<0.05)$, but decreased at $480 \mathrm{~d}$ of age $(p<0.05)$, in $p 21^{-/-}$mice relative to $p 21^{+/+}$.

age, $p 21^{-/-}$mice have an increase in the number of cells in the subependyma that retain BrdU labeling for $30 \mathrm{~d}$ (Fig. 1C). Conversely, at $480 \mathrm{~d}$ of age, $p 21^{-/-}$mice have a decrease in the number of cells in the subependyma that retain BrdU labeling for $30 \mathrm{~d}$ (Fig. 1C). Although the BrdU-retaining cells in the present experiments have not been further characterized, BrdU-retaining cells have been shown to continue to proliferate in vivo (Maslov et al. 2004). Nevertheless, together the data from the neurosphere assay in vitro and long-term retention of BrdU in vivo both indicate that loss of p21 function results in an increase in NSCs in young adults that is followed by an acceleration in the progressive decline in the NSC population during aging. The cellular mechanisms of these effects are investigated below.

Proliferation rate in vitro is increased in NSCs from young $\mathrm{p} 21^{-/-}$mice but decreased in NSCs from old $\mathrm{p} 21^{-/-}$mice

Loss of p21 altered in vitro NSC proliferation rate in an age-dependent fashion corresponding to the changes in 
in vivo NSC numbers as revealed by the cumulative BrdU labeling in vitro, cell number expansion, neurosphere size, and expansion of neurosphere numbers in primary cultures (Figs. 2A-C and $3 \mathrm{~A}$, respectively). At ages when $p 21^{-/-}$NSC numbers are increased, primary p21 $1^{-/-}$neurosphere cultures proliferate faster: higher numbers of BrdU labeled cells, more total cells, larger neurospheres /reflecting more asymmetrical divisions
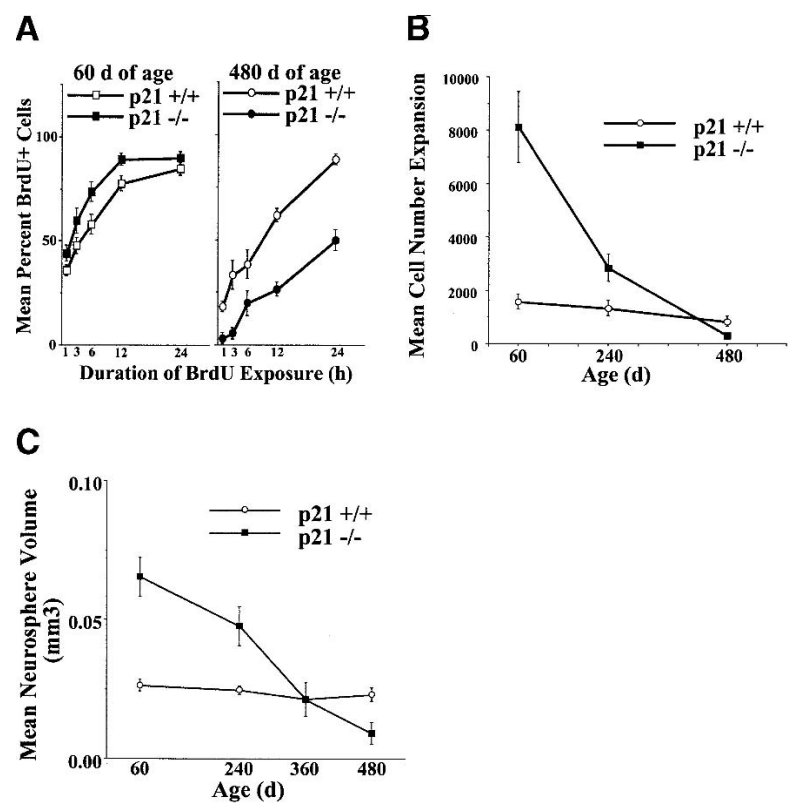

Figure 2. p21 loss in vivo results in neural stem cell expansion and reduced longevity mediated by an initial decrease and subsequent increase in cell cycle time, respectively, as measured by BrdU labeling in vitro, cell expansion, and clonal neurosphere size. (A) Cumulative BrdU labeling of primary neurosphere cultures from $p 21^{+/+}$and $p 21^{-/-}$mice at $60 \mathrm{~d}$ (left panel) and $480 \mathrm{~d}$ of age (right panel). Data are presented as mean \pm SEM ( $n=3-4$ per genotype per age per BrdU exposure time). There was a significant interaction between genotype and age $[\mathrm{F}(1,81)=57.74$, $p<0.05]$ with BrdU labeling in a higher proportion of cells in primary neurospheres from $60-\mathrm{d} p 21^{-/-}$mice $(p<0.05)$, but in a lower proportion of cells in primary neurospheres from 480-d $p 21^{-/-}$mice $(p<0.05)$, relative to $p 21^{+/+}$mice. Regression analyses of the BrdU incorporation data produced cell cycle estimates of $17.71 \mathrm{~h}$ for $p 21^{+/+}$at $60 \mathrm{~d}, 14.27 \mathrm{~h}$ for $p 21^{-/-}$at $60 \mathrm{~d}, 20.21 \mathrm{~h}$ for $p 21^{+/+}$at $480 \mathrm{~d}$, and $43.80 \mathrm{~h}$ for $p 21^{-/-}$at $480 \mathrm{~d}$. $(B)$ Cell expansion (total number of cells at end of culture divided by initial number of cells; presented as mean \pm SEM) in primary neurosphere cultures from $p 21^{+/+}$and $p 21^{-/-}$mice at 60, 240, and $480 \mathrm{~d}$ of age $(n=5-8$ per group per age). There was a significant genotype by age interaction $[\mathrm{F}(2,40)=10.08, p<0.05]$. Cell number expansion is increased at 60 and $240 \mathrm{~d}$ of age $(p s<0.05)$, but decreased at $480 \mathrm{~d}$ of age $(p<0.05)$, in $p 21^{-/-}$neurosphere cultures relative to $p 21^{+/+}$. (C) Volumes (mean \pm SEM) of primary neurospheres derived from adult $p 21^{-/-}$and $p 21^{+/+}$mice at 60-480 d of age ( $n=5-8$ per group per age). There was a significant genotype by age interaction $[\mathrm{F}(3,42)=4.84, p<0.05]$. Relative to $p 21^{+/+}$, primary $p 21^{-/-}$neurospheres were significantly larger than $p 21^{+/+}$neurospheres derived from mice at 60 and 240 $\mathrm{d}(p s<0.05)$, whereas primary $p 21^{-/-}$neurospheres were significantly smaller than $p 21^{+/+}$from mice at $480 \mathrm{~d}(p<0.05)$. producing more progenitors), and more neurospheres (reflecting more symmetric divisions). Whereas, at ages when $p 21^{-/-}$NSC numbers are decreased, primary $p 21^{-/-}$ neurosphere cultures proliferate slower: lower numbers of BrdU labeled cells, fewer total cells, smaller neurospheres (reflecting fewer asymmetrical divisions), and fewer neurospheres (reflecting fewer symmetric divisions).

Analyses of in vitro BrdU labeling of neurospheres from $p 21^{+/+}$and $p 21^{-/-}$at $60 \mathrm{~d}$ and $480 \mathrm{~d}$ (Fig. 2A) revealed an age-dependent effect of p21 loss. More neurosphere cells derived from $p 21^{-/-}$mice at $60 \mathrm{~d}$ incorporated BrdU across exposure lengths than did cells from p $21^{+/+}$mice at $60 \mathrm{~d}$, and, conversely, fewer neurosphere cells derived from $p 21^{-1-}$ mice at $480 \mathrm{~d}$ incorporated BrdU across exposure lengths than did cells from $p 21^{+/+}$ mice at $480 \mathrm{~d}$. Regression analyses of BrdU labeling data indicated that the average cell cycle times for neurosphere cells from $p 21^{+/+}$mice at $60 \mathrm{~d}$ is $17.71 \mathrm{~h}$, from p $21^{-/-}$mice at $60 \mathrm{~d}$ is $14.27 \mathrm{~h}$, from $p 21^{+/+}$mice at $480 \mathrm{~d}$ is $20.21 \mathrm{~h}$, and from $p 21^{-1-}$ mice at $480 \mathrm{~d}$ is $43.80 \mathrm{~h}$. Thus, it appears that $p 21^{-1-}$ NSCs undergo dramatic ( 2.9-fold) lengthening of cell cycle times during aging, whereas p21 $1^{+/+}$NSCs show only a modest $(\sim 1.1$-fold $)$ increase in cell cycle time during the same period. Similarly, analyses of cell expansion during primary neurosphere cultures (Fig. 2B) revealed an interaction between age and genotype. Primary neurosphere cultures derived from p21 $1^{-/-}$mice at $60 \mathrm{~d}$ and $240 \mathrm{~d}$ contained more cells than cultures from $p 21^{+/+}$mice of the same ages, whereas primary neurosphere cultures derived from $p 21^{-/-}$mice at $480 \mathrm{~d}$ contained fewer cells than cultures from $\mathrm{p}^{21^{+/+}}$ mice of the same age. Furthermore, p21 loss altered primary neurosphere size in an age-dependent fashion, demonstrating that the changes in proliferation rate are observable at the clonal (i.e., individual neurosphere) level of NSC proliferation (Fig. 2C). Primary $p 21^{-/-}$neurospheres were significantly larger than $p 21^{+/+}$neurospheres derived from mice at $60 \mathrm{~d}$ and $240 \mathrm{~d}$, whereas primary $p 21^{-/-}$neurospheres were significantly smaller than $p 21^{+/+}$neurospheres derived from mice at $480 \mathrm{~d}$. In contrast, analyses of individual cell diameters by flow cytometry failed to reveal significant differences between cells from early and late passage $p 21^{+/+}$and $p 21^{-/-}$ neurosphere cultures (see below), suggesting that differences in cell size do not contribute to differences in neurosphere size.

Moreover, p21 loss also altered the total number of NSC symmetric divisions during primary cultures in an age-dependent fashion as revealed by the total number of secondary neurospheres derived during bulk passaging (Fig. 3A). Bulk passaging of primary neurosphere cultures from $60 \mathrm{~d}$ or $480 \mathrm{~d} \mathrm{p} 21^{-/-}$mice resulted in significantly more or significantly fewer, respectively, total secondary neurospheres relative to cultures from $p 21^{+/+}$mice of the same age. In contrast, passaging of single primary neurospheres of the same size (and thus, containing approximately the same number of cells) did not result in a significant effect of $\mathrm{p} 21$ loss on the number of secondary neurospheres (Fig. 3B). Together these findings demon- 
A

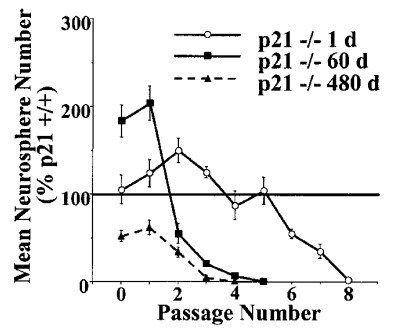

B

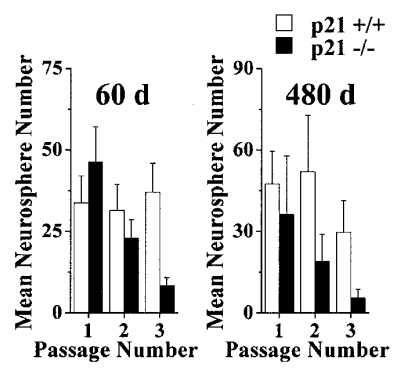

Figure 3. p21 loss increases the expansion but then decreases the maintenance of self-renewal of neural stem cells in vitro. $(A)$ Neurosphere expansion and self-renewal during bulk (10 cell/ $\mu \mathrm{L}$ in $500 \mu \mathrm{L}$ ) passaging in vitro. Data presented as mean \pm SEM. p21 $1^{-/-}$neurospheres from post-natal 1 and $60 \mathrm{~d}$ of age show an initial increase in expansion, whereas $p 21^{-/-}$neurospheres from post-natal $480 \mathrm{~d}$ show an initial decrease in expansion compared to $p 21^{+/+}$neurospheres. Moreover, $p 21^{-/-}$neurospheres from all ages show eventual self-renewal exhaustion. For cultures derived from mice at $1 \mathrm{~d}$ of age, there was a significant interaction between genotype and passage number $[\mathrm{F}(8,80)=4.31, p<0.05]$ with increased neurosphere numbers in $p 21^{-/-}$cultures on passages 1,2 , and 3 (ps $<0.05$ ), but decreased neurosphere numbers on passages 6,7 , and $8(p s<0.05)$ relative to $p 21^{+/+}$cultures. Similarly, for cultures derived from mice at $60 \mathrm{~d}$ of age, there was a significant interaction between genotype and passage number $[\mathrm{F}(5,50)=17.90, p<0.05]$ with increased neurosphere numbers in $p 21^{-/-}$cultures on primary and passage 1 ( $\left.p s<0.05\right)$, but decreased neurosphere numbers on passages 3,4 , and 5 ( $p s<0.05)$ relative to $p 21^{+/+}$cultures. Whereas for cultures derived from mice at $480 \mathrm{~d}$ of age, there was a significant main effect of genotype $[\mathrm{F}(1,8)=248.85, p<0.05]$ with decreased neurosphere numbers in $p 21^{-/-}$cultures on primary and all passages relative to $p 21^{+/+}$cultures $(p s<0.05)$. (B) Neurosphere expansion and self-renewal during the passaging of single neurospheres (of the same size) in vitro. Data presented as mean \pm SEM. No effects are observed on primary passaging of single neurospheres from 60 or $480 \mathrm{~d}$ of age, but neurospheres from both ages show eventual self-renewal exhaustion. For neurospheres derived from mice at $60 \mathrm{~d}$ of age, there was a significant interaction between genotype and passage number [F $(2$, $56)=3.66, p<0.05]$ with decreased secondary neurosphere numbers in $p 21^{-/}$cultures on passage $3(p<0.05)$; thereafter, too few $p 21^{-/}$of appropriate size were available for single neurosphere passaging. Similarly, for neurospheres derived from mice at $480 \mathrm{~d}$ of age, there was a significant effect of genotype [F $(1,15)=4.92, p<0.05]$ with decreased secondary neurosphere numbers in $p 21^{-/-}$cultures on passages 2 and $3(p<0.05)$; thereafter, too few $p 21^{-/-}$of appropriate size were available for single neurosphere passaging. strate that $\mathrm{p} 21$ loss alters the total number of symmetric divisions that individual NSCs undergo in an age-dependent fashion, but does not appear to bias the mode of division to either symmetric or asymmetric divisions.

Accordingly, $p 21^{-/-}$NSCs from young adults 160 and $240 \mathrm{~d}$ of age) proliferate faster than $\mathrm{p} 21^{+/+}$; thus, the increase in the total number of in vivo NSC symmetric divisions (and, indeed, all cell divisions) from $p 21^{-/-}$ mice relative to $p 21^{+/+}$mice can be accounted for by shorter cell cycle times. In contrast, $p 21^{-/-}$NSCs from old mice (480 d of age) proliferate more slowly than $\mathrm{p}^{21^{+/+}}$, suggesting that NSC loss during aging is due to lengthening of cell cycle time.

\section{Loss of p21 increases NSC proliferation in vitro and reveals NSC exhaustion}

The decrement in NSC longevity in the aged $p 21^{-/-}$mice in vivo along with the age-dependent changes in proliferation kinetics observed during primary neurosphere cultures suggest that the increased proliferation (i.e., disruption of relative quiescence) due to loss of p21 causes a subsequent reduction in the ability of NSCs to selfrenew for the lifetime of the organism (i.e., loss of longevity). This result predicts that $p 21^{-/-}$NSCs forced to divide repeatedly in vitro would show diminished longevity (i.e., limited self-renewal) relative to $p 21^{+/+}$NSCs. Furthermore, it also predicts that older $p 21^{-/-}$NSCs should show less longevity than younger $p 21^{-/-}$NSCs. In order to test these hypotheses, we performed serial passaging of neurosphere cultures in bulk at a constant clonal density (Morshead et al. 2003) and serial passaging of single neurospheres of equivalent size (and therefore equivalent cell number) to determine the expansion capacity of neurosphere cultures derived from young and old $p 21^{-/-}$and $p 21^{+/+}$mice. During bulk passaging, $p 21^{-/-}$ neurosphere cultures from all ages displayed reduced self-renewal compared to $p 21^{+/+}$neurosphere cultures and eventual complete exhaustion of the self-renewal capacity with neurosphere cultures from older $p 21^{-/-}$ mice exhausting more quickly than those from younger p21 $1^{-/-}$mice (Fig. 3A). Similarly, serial passaging of individual neurospheres from $60 \mathrm{~d}$ and $480 \mathrm{~d}$ mice also revealed exhaustion of $p 21^{-/-}$neurospheres (Fig. 3B), demonstrating that exhaustion is observable at the level of a single neurosphere-forming cell. Neurosphere culture exhaustion was also observed during experiments examining bulk passaging at a higher clonal cell density $(20$ cells $/ \mu \mathrm{L})$, and from experiments in which neurospheres were isolated and passaged in either EGF alone or FGF and heparin (data not shown). Thus, the neurosphere culture exhaustion in the absence of $\mathrm{p} 21$ is not specific to a particular age, an effect of culture density, or an effect of specific growth factor responsiveness. These findings indicate that the reduced longevity of $p 21^{-/-}$neurosphere cultures is unlikely to reflect a lack of optimal culture conditions as has been shown to be critical for the longterm maintenance of glia progenitor cell lines in vitro (Mathon et al. 2001; Tang et al. 2001). Together, these findings demonstrate that the decrease in NSC longevity 
in the absence of $\mathrm{p} 21$ is due to a reduction in the longterm self-renewal ability of individual NSCs.

NSC exhaustion in vitro in the absence of p21

is accompanied by progressive decline

in proliferation rates

Similar to the effects observed in p21-deficient primary neurosphere cultures, analyses of cumulative BrdU labeling, cell number expansion, and neurosphere size during in vitro passaging revealed dynamic changes in NSC proliferation rate as a function of age and passage. The exhaustion of $p 21^{-/-}$neurosphere cultures are paralleled by a decrease in the rate of cumulative BrdU labeling in cultures derived from $p 21^{-/-}$at $60 \mathrm{~d}$ of age. In $p 21^{-/-}$ neurospheres cultures, a significant decrease in the rate of BrdU incorporation was observed between primary and following the second passage (i.e., tertiary culture), whereas no change was observed for $p 21^{+/+}$neurosphere cultures between primary and following the second passage (Fig. 4A). Regression analyses of the BrdU labeling curves revealed that $p 21^{-/-}$neurosphere cells undergo an average twofold increase in cell cycle time between primary $(14.27 \mathrm{~h})$ and passage $2(28.86 \mathrm{~h})$, whereas $p 21^{+/+}$ neurosphere cells undergo only a 1.12 -fold increase in cell cycle time between primary $(17.71)$ and passage 2 (19.89). Thus, in vitro proliferative exhaustion in $p 21^{-1-}$ neurosphere cultures is accompanied by progressively longer cell cycles.

Similarly, $p 21^{-/-}$neurosphere cultures from young mice ( 1 or $60 \mathrm{~d}$ of age) initially displayed increased cell number expansion relative to $\mathrm{p}^{2} 1^{+/+}$cultures, followed by progressive decreases in cell number expansion leading to exhaustion; $p 21^{-/-}$neurosphere cultures from old mice $(480$ d) had initially decreased cell number expansion relative to $p 21^{+/+}$cultures and then further decreased leading to exhaustion (Fig. 4B). Furthermore, individual $p 21^{-/-}$neurosphere volumes decreased progressively by $97 \%$ during passaging, whereas $p 21^{+/+}$ neurosphere volumes did not change significantly during passaging (Fig. 4C). Conversely, flow cytometry revealed no differences in the size of individual $p 21^{+/+}$and $p 21^{-/-}$ cells in neurosphere cultures at passage 1 or passage 4 (Fig. 4D). These findings demonstrate that changes in p21 $1^{-/}$neurosphere culture exhaustion is accompanied by a decline in proliferation rate during bulk passaging, and these effects are detectable at the clonal (i.e., individual neurosphere) level.

\section{Cell death is not increased during NSC exhaustion in vitro due to p21 loss}

The contribution of cell death to $p 21^{-/-}$NSC exhaustion was assessed by quantifying the proportion of dead cells using trypan blue staining of nonviable cells in dissociated neurospheres during passaging. At each passage, neurosphere cultures contained a minority of nonviable cells, but no differences were detected between the $p 21^{+/+}$and $p 21^{-/-}$cultures (Fig. 4E). This finding demon- strates that NSC exhaustion is not associated with an increase in dying cells. In addition, staining for markers of apoptosis (DNA strand breaks or exposed membrane phospholipid phosphatidylserines) of undissociated neurospheres plated on MATRIGEL at various passages failed to detect apoptotic cells in either $p 21^{+/+}$or $p 21^{-/-}$ neurospheres (data not shown), suggesting that programmed cell death is unable to account for either the initial increase or later decrease in cell numbers in $p 21^{-/-}$ neurospheres.

Loss of p21 does not alter multipotentiality or change the lineage fate of NSCs

Cells from primary neurospheres from $p 21^{-/-}$and $p 21^{+/+}$ mice at all ages differentiated into neurons and glia, indicating that the decrement in NSC self-renewal in vivo is not accompanied by a reduction in multipotentiality (data not shown). Furthermore, the loss of self-renewal in p21 $1^{-/-}$neurosphere cultures derived at $60 \mathrm{~d}$ of age during passaging was not associated with a loss of multipotentiality or changes in the proportion of neural or glial progeny when neurospheres were differentiated in serum (Fig. 5A). Similarly, qualitative analyses of passaged p $21^{+/+}$and $p 21^{-/-}$neurospheres derived from mice at 480 $\mathrm{d}$ revealed that multipotentiality was maintained in all neurospheres examined until at least the third passage; thereafter, insufficient numbers of appropriately sized $p 21^{-/-}$neurospheres were available. These findings demonstrate that during constant clonal neurosphere cultures, $p 21^{-/-}$NSCs displayed a specific reduction in the maintenance of self-renewal without loss of multipotentiality. Furthermore, the finding that there was no increase in the proportions of neurons and glia suggests that NSCs did not change fates by differentiating into progenitors, as is seen after Notch pathway mutations (Hitoshi et al. 2002).

Loss of p21 does not directly affect NSC progeny

in vivo, but stem cell exhaustion results

in accelerated declines in neural progenitors

and neurogenesis during aging

NSCs comprise a small minority of the total cells in a neurosphere, with the majority of cells having either little self-renewal ability, limited lineage potency, or both (i.e., restricted progenitor cells) (Morshead et al. 1998; Hitoshi et al. 2002). It is unclear whether the effects of p21 loss on cell number in neurosphere cultures are due to changes in NSC or progenitor cell populations. Given that progenitor cells comprise the majority of cells in neurospheres, the changes in neurosphere size cannot be due to the changes in number of NSCs alone. However, the high correlation between changes in neurosphere size and changes in self-renewal suggests that a common mechanism underlies both of these effects. In order to test for a direct effect of p21 loss on progenitor cell populations, we examined the expression of the endogenous proliferation marker, Ki67 (Giardino et al. 2000; Enwere et al. 2004), in the adult subependyma of 
A

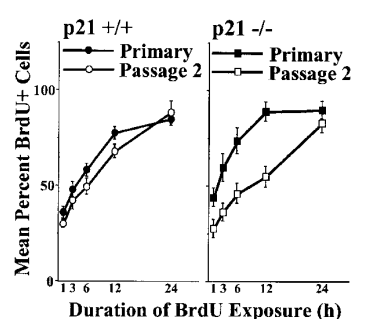

C

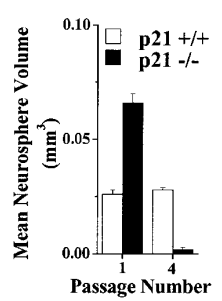

D

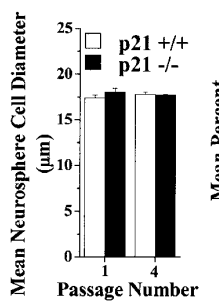

B

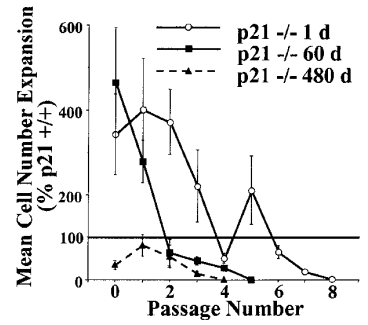

E

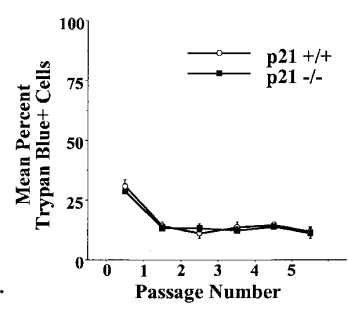

Figure 4. $p 21^{-/-}$neurosphere culture expansion and exhaustion in vitro are accompanied by an initial decrease and a subsequent increase in cell cycle time, respectively, as measured by BrdU lableing in vitro, cell expansion, and clonal neurosphere size, whereas there is no change in individual cell size or cell viability. (A) Cumulative BrdU labeling of primary and twicebulk-passaged neurosphere cultures from $\mathrm{p} 21^{+/+}$(right panel) and $p 21^{-1-}$ (left panel) mice at $60 \mathrm{~d}$ of age. Data are presented as mean \pm SEM. There was a significant interaction between genotype and passage $[\mathrm{F}(1,96)=4.46, p<0.05]$ with $p 21^{-/-}$cells in primary culture incorporating BrdU at a faster rate than $p 21^{-/-}$ cells after passage $2(p<0.05)$; conversely, $p 21^{+/+}$cells did not differ in BrdU incorporation rate between primary and passage 2 $(p>0.05)$. Regression analyses of the BrdU incorporation data produced cell cycle estimates of $17.71 \mathrm{~h}$ for $\mathrm{p}^{2} 1^{+/+}$primary neurospheres, $19.89 \mathrm{~h}$ for $p 21^{+/+}$passage 2 neurospheres, $14.27 \mathrm{~h}$ for p21 $1^{-/-}$primary neurospheres, and $28.89 \mathrm{~h}$ for $p 21^{-/-}$passage 2 neurospheres. (B) Cell number expansion (mean \pm SEM) during bulk passaging of neurosphere cultures from $p 21^{+/+}$and $p 21^{-/-}$ mice at 1,60 , and $480 \mathrm{~d}$ of age. $p 21^{-/-}$neurospheres from postnatal 1 and $60 \mathrm{~d}$ of age is initially larger but subsequently smaller than $p 21^{+/+}$neurospheres, demonstrating a significant correlation between neural stem cell self-renewal and total progeny number in vitro. For cultures derived from mice at $1 \mathrm{~d}$ of age, there was a significant interaction between genotype and passage number $[F(8,80)=3.30, p<0.05]$ with increased cell number expansion in $p 21^{-/-}$cultures on primary and passages 1,2 , 3, and 5 $(p s<0.05)$, but decreased cell number expansion in $p 21^{-/-}$cultures on passages $4,6,7$, and $8(p s<0.05)$ relative to $p 21^{+/+}$cultures. Similarly, for cultures derived from mice at $60 \mathrm{~d}$ of age, there was a significant interaction between genotype and passage number $[\mathrm{F}(5,50)=9.35, p<0.05]$ with increased cell number expansion in $p 21^{-/-}$culture on primary culture and passages $1(p s<0.05)$, but decreased cell number expansion in $p 21^{-/-}$cultures on passages 3,4 , and 5 ( $\left.p s<0.05\right)$ relative to $p 21^{+/+}$cultures; whereas for cultures derived from mice at $480 \mathrm{~d}$ of age, there was a significant main effect of genotype $[\mathrm{F}(1,8)=15.22, p<0.05]$ with decreased cell number expansion in $p 21^{-/-}$cultures on primary and all passages relative to $p 21^{+/+}$cultures $(p s<0.05)$. (C) Volumes (mean \pm SEM) of passage 1 and passage 4 neurospheres derived from adult $p 21^{-/-}$and $p 21^{+/+}$mice at $60 \mathrm{~d}$. There was a significant genotype by passage interaction $[\mathrm{F}(1,16)=9.10, p<0.05]$ with $p 21^{-/-}$neurospheres larger in passage 1 and smaller in passage 4 than $p 21^{+/+}$neurospheres. $(D)$ Diameters (mean \pm SEM) of individual cells in passage 1 and passage 4 neurosphere cultures from $p 21^{+/+}$and $p 21^{-/-}$at $60 \mathrm{~d}$ of age. No significant effects were observed. $(E)$ Proportions (mean \pm SEM) of nonviable cells in primary and bulk-passaged neurosphere cultures from $p 21^{+/+}$ and $p 21^{-/-}$mice at $60 \mathrm{~d}$ of age. No effect of $\mathrm{p} 21$ loss was detected. However, there was a significant effect of passage $[\mathrm{F}(5,64)=24.49$, $p<0.05$ ] with primary cultures containing more dead cells than from all passaged cultures $(p s<0.05)$.

the lateral ventricle of $p 21^{-/-}$and $p 21^{+/+}$mice at $60 \mathrm{~d}$ and $480 \mathrm{~d}$ of age. In contrast with the effect of 21 loss on NSC number, there was no effect of $\mathrm{p} 21$ loss on the number of Ki67-labeled cells in 60-d mice, but there was a significantly greater age-related reduction in the number of Ki67-labeled cells in $p 21^{-/-}$compared to $p 21^{+/+}$mice at $480 \mathrm{~d}$ (Fig. 5B). Similarly, quantification of BrdU-labeled cells $1 \mathrm{~h}$ post-labeling in $\mathrm{p} 21^{+/+}$and $p 21^{-/-}$mice at $60 \mathrm{~d}$ indicated that progenitor numbers were not altered by loss of p21 (data not shown). These findings indicate an apparent lack of direct effect of p21 loss on progenitor cell populations in young adult mice, suggesting that changes in the number of cells in a neurosphere are mediated by primary effects on NSCs. However, the number of progenitors in vivo declines proportionally to the decline in the number of NSCs.

The in vivo consequences of p21 loss in young adult mice and ensuing NSC depletion in old adult mice for neurogenesis in the olfactory bulb also were examined. Consistent with the finding that loss of p21 did not significantly alter the number of rapidly proliferating progenitors in the subependyma, there were no significant differences in the number of new neurons $\left(\mathrm{BrdU}^{+}\right)$ $\left.\mathrm{NeuN}^{+}\right)$and new nonneuronal cells $\left(\mathrm{BrdU}^{+} / \mathrm{NeuN}^{-}\right)$; it is unclear whether $\mathrm{NeuN}^{-}$cells are immature neurons not yet expressing NeuN or other differentiated nonneuronal cells) in the granule cell layer of the olfactory bulb between $p 21^{+/+}$and $p 21^{-/-}$mice at $60 \mathrm{~d}$ of age (Fig. 5C). Conversely, the numbers of new neurons and new nonneuronal cells in the granule cell layer of the olfactory bulb were substantially reduced in mice at $480 \mathrm{~d}$ of age regardless of genotype; moreover, these declines were significantly greater in the $p 21^{-/-}$mice relative to $p 21^{+/+}$ mice (Fig. 5C). These findings demonstrate that loss of p21 does not directly alter the survival or differentiation capacity of the progeny of NSCs in vivo in young (60 d of age) adult mice, but that the accelerated loss of NSCs observed in p21-deficient mice results in substantially fewer new differentiated cells in the olfactory bulb of aging (480 d of age) mice.

\section{Discussion}

Our results (summarized in Table 1) demonstrate that p21 plays a significant role in regulating NSC proliferation and indirectly their longevity. In two quantitative assays of forebrain NSC numbers in vivo, p21 loss in young (60-240 d of age) adult mice resulted in an increase in forebrain subependyma-derived clonal neurospheres and an increase in long-term BrdU-retaining 
Consistent with the present findings, Qiu et al. (2004) reported that brain ischemia induces higher proliferation of $p 21^{-/-}$NSCs relative to $p 21^{+/+}$in young adult mice. Conversely, their report did not reveal any difference between $p 21^{+/+}$and $p 21^{-/-}$NSC numbers under normal conditions. Qiu et al. did not assess any of the other measures of $p 21^{-/-}$NSC numbers or proliferation (i.e., BrdU retention or measures of proliferation in vitro that were used in the present study), making full comparison between studies difficult. However, two procedural differences may be responsible for this apparent discrepancy. First, Qiu et al. used relatively high-density culture conditions that are nonclonal (see Supplemental Material) and thus, of low sensitivity to differences in numbers of NSCs (e.g., see Morshead and van der Kooy 2004). Second, Qiu et al. used transgenic mice on a pure 129/SV background, whereas we used transgenic mice on a mixed B6c57 and 129/SV background. Thus, differences in background genetics may interact with p21 loss in the regulation of NSC proliferation. Importantly however, the in vivo exhaustion phenomenon observed in the present report does not occur in NSCs from either pure background in the presence of p21 (T.E. Kippin and D. van der Kooy, unpubl.). Nevertheless, $p 21^{-/-}$NSCs proliferation was increased under specific conditions in both studies, and, thus, shorter cell cycle times can account for the increase in NSC expansion both in vivo and in vitro observed in the present study.

Subsequently, old (480 d of age) mice lacking p21 have a decrease in forebrain subependyma-derived clonal neurospheres and a decrease in long-term BrdU-retaining cells in forebrain subependyma in vivo. A similar pattern of increased expansion followed by reduced self-renewal of NSCs in the absence of p21 also occurred during neurosphere passaging in vitro. BrdU-labeling in vitro revealed that loss of NSC self-renewal during passaging is accompanied by a decrease in proliferation rate. Furthermore, NSC numbers and total cell numbers in the neurospheres were highly correlated during passaging, demonstrating that the numbers of symmetric (i.e., producing two NSCs) and asymmetric (i.e., producing one NSC and one progenitor) divisions are affected in a similar way by $\mathrm{p} 21$ loss in vitro. These results indicate that the primary effect on NSCs of p21 loss is a decrease in cycle time, leading to NSC expansion due to more symmetric divisions. Accordingly, $p 21^{-/-}$NSCs ultimately go through so many divisions that they exhaust their selfrenewal potential and subsequently enter senescence, resulting in an accelerated decline in the NSC population and neurogenesis in the olfactory bulb during aging. Thus, NSCs appear to have limited self-renewal (i.e., can go through only a finite number of divisions) that is normally conserved by relative quiescence, such that at least a population of NSCs are maintained throughout the lifetime of the organism.

\section{Loss of p21 specifically increases symmetric and asymmetric divisions of NSCs}

The present findings demonstrate that 21 has a specific role in negatively regulating the proliferation of NSCs.
However, several findings demonstrate that $\mathrm{p} 21$ does not negatively regulate all cell proliferation in the brain. First, the progenitor cell population in the adult subependyma was not affected by p21 loss. Second, neurosphere cell numbers during passaging were highly correlated with self-renewal capacity following p21 loss, suggesting that a primary NSC effect is responsible for changes in total cell numbers within a neurosphere (i.e., progenitor cell numbers are only indirectly influenced by p21 loss). Accordingly, we propose that p21 has a specific role in the maintenance of adult NSC relative quiescence (Morshead et al. 1994): p21 loss increases the number of divisions as a direct result of shorter cell cycles leading to the expansion of the NSC population in vivo and in vitro. Normally, p21 inhibits cell cycle progression into $S$ phase via interactions with both cyclin E-CDK2 complex and PCNA; thus p21 loss directly shortens the cell cycle (Vidal and Koff 2000). Furthermore, p21 not only contributes to NSC relative quiescence in vivo, but also inhibits NSC proliferation in vitro under optimal conditions.

In contrast to the early expansion of NSCs in vivo and neural progenitors (in neurospheres) in vitro, the numbers of their rapidly proliferating progeny (i.e., progenitors) in the adult forebrain subependyma are not affected by 21 loss in vivo. Two mechanisms may account for the differences between the effect of p21 loss on progenitor cells in vivo and in vitro. First, the high correlation between NSC symmetric and total divisions in our in vitro data suggests that the lack of adult forebrain progenitor expansion following p21 loss in vivo may be regulated by a $\mathrm{p} 21$-independent cell cycle mechanism in a cell-nonautonomous manner (i.e., via cell-cell interactions) that is present in the adult brain but not in neurospheres. Second, given that cell death and migration are the predominant post-mitotic fates for progenitors in the adult subependyma (Morshead and van der Kooy 1992; Luskin 1993; Lois and Alvarez-Buylla 1994; Morshead et al. 1998), an increase in progenitor cells produced by increased NSC proliferation could be compensated by either increased cell death, increased migration out of the subependyma, or failure to migrate in the absence of p21 in vivo. However, the number of adult-born olfactory bulb cells was not increased in young adult $p 21^{-/-}$ mice, demonstrating that the number of surviving and differentiating progeny of NSCs is maintained at a constant level in the absence of p21.

\section{Loss of p21 exhausts NSC self-renewal}

Following NSC expansion in young adults, $p 21^{-/-}$mice displayed an accelerated reduction of NSCs at $480 \mathrm{~d}$, thus demonstrating a decrement in in vivo longevity of NSCs. Furthermore, in vitro isolated $p 21^{-1-}$ NSCs display initially increased expansion followed by a progressive reduction in self-renewal and eventual exhaustion (i.e., loss of neurosphere-forming ability) in vitro. Importantly, the finding that the neurosphere-forming cells that eventually undergo exhaustion are initially capable 
of high levels of self-renewal and are multipotential demonstrates that these spheres are not generated from progenitor cells because progenitor-derived spheres lack self-renewal and are not capable of forming neurons (Seaberg and van der Kooy 2002; Seaberg et al. 2005). Together, these findings indicate that the p21 loss results in NSC self-renewal exhaustion, as a consequence of prior increased division either in vivo or in vitro. Thus, the increase in NSC division induced by p21 loss has allowed the demonstration of NSC proliferative exhaustion; essentially, NSCs do not have an unlimited self-renewal capacity in the absence of transformation (Morshead et al. 2002).

Decreased NSC self-renewal also was associated with a progressive increase in cell cycle time (i.e., slower proliferation rate), indicating that NSC exhaustion may be mediated by proliferative senescence. Cell division in $p 21^{-/-}$NSCs may lead to senescence by several distinct mechanisms. First, several p21-independent cell senescence pathways have been elucidated, including cell cycle arrest via the $\mathrm{p} 16^{\mathrm{INK} 4 \mathrm{a}}$-retinoblastoma tumor suppressor protein pathway and the PTEN-p2 $7^{\text {Kip } 1}$ pathway (Bringold and Serrano 2000). Moreover, cell expansion results in the progressive up-regulation of $\mathrm{p} 16^{\text {INKa }}$ (Serrano 1997) and $\mathrm{p} 27^{\mathrm{Kip} 1}$ (Wagner et al. 2001); thus, the absence of p21 may lead to NSC senescence (due to prior increased proliferation) mediated by increased expression of other cell cycle inhibitors. However, using immunocytochemistry, we failed to detect expression of the putative senescence marker, $\beta$-galactosidase (e.g., Tang et al. 2001) in $p 21^{-/-}$NSCs undergoing exhaustion (T.E. Kippin and D. van der Kooy, unpubl.).

Second, decreased NSC self-renewal after Notch pathway mutations has been associated previously with increased differentiation into neurons and glia, suggesting terminal symmetric division (i.e., producing two progenitors) as a mechanism of NSC loss (Hitoshi et al. 2002). However, $p 21^{-/-}$NSCs do not yield more neurons or glia when neurospheres were differentiated in vitro from either primary cultures or from subsequent passages. Thus, it is unlikely that terminal symmetric divisions into two progenitor cells and later differentiation contribute to the decline in $p 21^{-/-}$NSCs seen in vivo or in vitro.

Third, decreased cell viability may mediate $p 21^{-/-}$ NSC exhaustion. p21 directs p53-mediated processes toward senescence over apoptosis, with p21 loss increasing apoptosis (Seoane et al. 2002). Cell division also increases p53 levels (Bringold and Serrano 2000). Thus, increased NSC division in the absence of $\mathrm{p} 21$ may elevate p53 and result in increased NSC death. However, no increase in cell death accompanied loss of self-renewal in $p 21^{-/-}$NSC cultures, indicating that increased apoptosis does not underlie NSC exhaustion. Furthermore, early in vitro passaging of $p 21^{-/-}$NSCs from young adult mice produce enlarged neurospheres, demonstrating that an increased rate of proliferation does not bias $p 21^{-/-}$cells unequivocally toward apoptosis. Similarly during embryonic development, p21 does not appear to regulate NSC expansion and NSC proliferation rate is at near maximum levels (Martens et al. 2000), yet the numbers of NSCs are increased in young adult $p 21^{-/-}$mice relative to $p 21^{+/+}$mice. Additionally, NSCs are highly resistant to apoptosis because of low expression of pro-apoptotic genes (Brazel et al. 2004). Together, these findings suggest a bias toward cell death is unlikely to explain the loss in NSC longevity.

Finally, cell division progressively decreases telomere length, and telomere shortening is linked to exhaustion of proliferative capacity (Harley et al. 1990; Hayflick 2000; Shay et al. 2000). The increased NSC proliferation in the absence of p21 observed in the present experiments may increase the rate of telomere shortening. Despite the presence of extremely long telomeres in mouse cells (Oulton and Harrington 2000), serial transplantations of mouse hematopoietic stem cells have revealed that telomere shortening may play a role in the senescence of these stem cells (Allsopp et al. 2001). Similarly, loss of telomerase function in TERC-deficient mice that are undergoing germline senescence is associated with reduced proliferation in adult-derived neurospheres, and importantly, the reduction in proliferation results in cell cycle arrest, but not cell death (Ferron et al. 2004). Accordingly, reductions in telomere length could potentially account for the effects of p21 loss on NSCs.

In summary, p21 loss results in decreased cell cycle times leading to an initial post-natal expansion of NSCs, demonstrating that $\mathrm{p} 21$ plays a role in the maintenance of relative quiescence in adult NSCs. The increase in NSC proliferation induced by $\mathrm{p} 21$ loss results in more total cumulative (i.e., both symmetric and asymmetric) cell divisions, leading to impairment of long-term selfrenewal and eventual exhaustion of NSCs in aging mice. Thus, a major implication of the present study is that adult multipotential NSCs have extensive, but finite, self-renewal capacity, and a population of these cells lasts the lifetime of an organism because their proliferation is tightly regulated (i.e., they are relatively quiescent). A similar role for p 21 has been demonstrated in hematopoietic stem cell regulation during serial repopulation (Cheng et al. 2000). Thus, stem cells in at least two tissues share the common properties of both finite proliferation capacity and p21-regulated relative quiescence as a mechanism to ensure maintenance of the stem cell niche throughout the life span. Furthermore, NSC self-renewal decrements result in a reduction in the production of new neurons in the olfactory bulb of aging mice.

\section{Materials and methods}

\section{Subjects}

All mice were derived from homozygous breeding of $p 21^{-/-}$ mice on a mixed B6129SF2/J background and their wild-type control strain $\left(p 21^{+/+} ; \mathrm{B} 6129 \mathrm{SF} 2 / \mathrm{J}\right)$, both purchased from Jackson Laboratories. For embryonic ages, time of pregnancy was determined by the first detection of a vaginal sperm plug by daily inspection. For all other ages, the day of birth was counted as post-natal day 0 , and animals were dissected at the appropriate 
ages. No obvious physical or anatomical abnormalities have been reported or presently observed in these mice.

\section{Neurosphere assay}

We used the in vitro neurosphere assay (Reynolds and Weiss 1996) as an index of the number of neural stem cells in vivo. Subsequently, the self-renewal and expansion characteristics of the neural stem cells in vitro was determined by serial passaging of neurospheres either in bulk or individually. And the differentiation characteristics of neural stem cell progeny in vitro were determined by culturing neurospheres in serum.

For ages E14 and post-natal $1 \mathrm{~d}$, the ganglionic eminence was dissected as described previously (Tropepe et al. 1999). For all subsequent ages, the subependyma of the lateral ventricle, which is the adult derivative of the ganglionic eminence, was dissected as described previously (Reynolds and Weiss 1996). Subependymal tissue was subjected to enzymatic digestion $(1.33 \mathrm{mg} / \mathrm{mL}$ trypsin, $0.67 \mathrm{mg} / \mathrm{mL}$ hyaluronidase, and $0.2 \mathrm{mg} /$ $\mathrm{mL}$ kynurenic acid) for $50 \mathrm{~min}$ at $37^{\circ} \mathrm{C}$, then isolated in serumfree media with trypsin inhibitor. Tissue from all ages was mechanically dissociated into a single cell suspension with a small-bore, fire-polished Pasteur pipette. Cell density and viability were determined using trypan blue exclusion.

Cells were cultured in the neurosphere assay (Reynolds and Weiss 1996) under clonal conditions (see Supplemental Material). Cells were plated at 10 cells $/ \mu \mathrm{L}$ in 24 -well $(0.5 \mathrm{~mL} /$ well $)$ uncoated plates (Nunclon) in serum-free medium containing 20 $\mathrm{ng} / \mathrm{mL}$ epidermal growth factor (EGF; mouse submaxillary; Sigma), $10 \mathrm{ng} / \mathrm{mL}$ fibroblast growth factor-2 (FGF; human recombinant; Sigma), and $2 \mu \mathrm{g} / \mathrm{mL}$ heparin (Upstate Biotech); independent cultures isolated in either EGF or FGF and heparin yielded the same pattern of results as combined growth factor conditions and are not presented here. The total number of spheres that formed in each well was counted after $7 \mathrm{~d}$; only colonies $>100 \mu \mathrm{m}$ in diameter were counted as spheres. Under these conditions it has been shown that neurosphere colonies are derived from single cells and serve as an index of the number of in vivo neural stem cells (Morshead et al. 2003). Neurosphere size was determined by measuring the diameters of individual neurospheres under light microscopy and expressed as a volume (assuming a spherical shape).

To determine the in vitro expansion and self-renewal capacity, we mechanically dissociated neurospheres into single cell suspension in bulk and recultured them under the same conditions as primary cultures, and the number of neurospheres was determined after $7 \mathrm{~d}$. Upon dissociation, the number of cells present at the end of each culture was calculated based on sample cell counts made with a hemocytometer under light microscopy and expressed as the average expansion from the initial starting population (i.e., total number of cells at end of culture divided by number of cells at start of culture). This was repeated up to a maximum of 10 passages; previous reports have demonstrated the neurospheres passaged up to 10 times (i.e., 10 wk in vitro) are virtually identical to primary cultures (Reynolds and Weiss 1996), but further passaging results in changes in proliferation and/or gene expression indicative of transformation events (Morshead et al. 2002). Separate single-sphere passaging experiments used spheres of the same diameter ( 200 $\mu \mathrm{m})$ and yielded the similar results as bulk passaging.

To determine multipotentiality, single neurospheres (of 200 $\mu \mathrm{m}$ diameter) were differentiated in 24-well plates coated with MATRIGEL basement membrane matrix $0.6 \mathrm{mg} / \mathrm{mL}$ in SFM; Becton-Dickinson) with $0.5 \mathrm{~mL}$ of SFM containing $1 \%$ fetal bovine serum (GIBCO). Wells were processed $7 \mathrm{~d}$ later using immunocytochemistry as described previously (Seaberg and van der Kooy 2002). We used anti- $\beta$-tubulin isotype III mouse monoclonal (IgG; 1:500; Sigma), anti-GFAP rabbit polyclonal (IgG; 1:400; Chemicon), FITC goat anti-rabbit (1:200; Jackson ImmunoResearch), and TRITC goat anti-mouse (1:200; Jackson ImmunoResearch). Cultures were counterlabeled with the nuclear stain Hoechst $33258(1 \mu \mathrm{g} / \mathrm{mL}$; Sigma). Fluorescence was visualized using a Nikon Microphot microscope, and the proportions of neurons and astrocytes generated were estimated by counting $\beta$-tubulin isotype $\mathrm{III}^{+}$and $\mathrm{GFAP}^{+}$cells as a percentage of Hoescht-positive nuclei in three random fields per differentiated neurosphere.

In vivo BrdU labeling, immunocytochemistry, and quantification

BrdU labeling of mitotic cells in S phase in vivo was used to index the number of neural stem cells in the subependyma of the lateral ventricle following long-term ( $30 \mathrm{~d})$ retention, the number of progenitor cells in the subependyma of the lateral ventricle following short-term $(1 \mathrm{~h})$ retention (Morshead et al. 1998; Hitoshi et al. 2002), and the number of adult-born neurons and glia in the olfactory bulbs following long-term (30 d) retention. Mice were injected with bromodeoxyuridine (BrdU, Sigma; $60 \mathrm{mg} / \mathrm{kg}$, i.p. in saline) every $3 \mathrm{~h}$ for five injections and sacrificed $1 \mathrm{~h}$ or $30 \mathrm{~d}$ after the final injection. Animals were overdosed with pentobarbital and perfused transcardially with PBS and $4 \%$ paraformaldehyde. Brains were removed and post-fixed overnight, cryprotected with $30 \%$ sucrose in PBS at $4{ }^{\circ} \mathrm{C}$, and then sectioned at $14 \mu \mathrm{m}$ thickness on a cryostat. Sections were incubated in $1 \mathrm{~N} \mathrm{HCl}$ at $60^{\circ} \mathrm{C}$ for $30 \mathrm{~min}$, then rinsed in PBS, and subsequently incubated in rat anti-BrdU antibody $(1: 100$; Seralab) at $4^{\circ} \mathrm{C}$ overnight, followed by FITC donkey anti-rat antibody (1:200; Jackson) for $2 \mathrm{~h}$. For long-term retention in the subependyma, the total number of BrdU-labeled cells was estimated using the optical dissector method (Coggeshall and Lekan 1996) in every eighth section (approximately every $100 \mu \mathrm{m}$ ) from the rostral tip of the crossing of the corpus callosum rostally and extending caudally to the rostral tip of the crossing of the anterior commissure. For short-term retention in the subependyma, the number of BrdU-labeled cells in a 1-mm section of the medial and lateral subependyma walls at the midportion of the ventricle were counted using an optical grid to give an estimate of the total number of cells in every eighth section for the same rostral-caudal region as above (Martens et al. 2002). For quantification of new cells in the adult olfactory bulb, the total number of BrdU-labeled cells in the granule cell layer of the main olfactory bulb was determined in every eighth section extending $\sim 1 \mathrm{~mm}$ anterior to the rostral portion of the accessory olfactory bulb; the number of new cells adopting a neuronal fate was determined by double-labeling with mouse anti-NeuN (1: 200; Chemicon) and visualized with Alexa Fluor 555 goat antimouse (1:300; Molecular Probes). To quantify the progenitor cell population in the subependyma, brain tissue from $p 21^{+/+}$ and $p 21^{-/-}$mice at $60 \mathrm{~d}$ and $480 \mathrm{~d}$ were treated with anti-Ki67 (1:500; Novocastra) and visualized with Alexa Fluor 568 goat anti-rabbit (1:300; Molecular Probes), and cells in the subependyma were counted in the same manner as for long-term BrdU labeling.

\section{In vitro BrdU labeling and cell cycle time estimate}

BrdU labeling of neurosphere cultures was used to estimate the cell cycle time of neural stem and progenitor cells in vitro. Neurospheres were prepared as above with the addition of BrdU $(1 \mu \mathrm{M})$ during the last $1,3,6,12$, or $24 \mathrm{~h}$ of culturing (i.e., all cells harvested at the same time, $7 \mathrm{~d}$ following the start of the 
culture). After the prescribed BrdU exposure, neurospheres were collected in bulk, dissociated into single cells, plated onto MATRIGEL-coated plates in SFM without growth factors for $1 \mathrm{~h}$, fixed with $4 \%$ paraformaldehyde for $20 \mathrm{~min}$, and washed with PBS. Cells were stained using the same procedure as above and counterlabeled with Hoechst. The proportion of BrdUlabeled nuclei was determined for neurosphere cultures derived from separate animals at ages and passages as indicated. Regression analyses of the active portion of each BrdU labeling curve (i.e., 1-12 h of BrdU exposure) were used to estimate cell cycle times assuming that all cells proliferate at the same rate and that every cell is labeled at the end of a single cycle.

\section{Detection of cell death}

The proportion of dead cells was determined using trypan blue viability cell counts at each passage for dissociated neurospheres from separate cultures derived from individual animals. Markers for apoptotic cells were assessed in undissociated neurospheres that were plated onto MATRIGEL-coated plates for 1 $\mathrm{h}$ prior to fixation. Apoptotic cells were labeled using the In Situ Cell Death Detection Kit, TMR Red (Roche) to label DNA strand breaks or Annexin V-FITC Apoptosis Detection Kit II (BD Biosciences) to label exposed phospholipid phosphatidylserines in cell membrane; positive controls for TMR red or Annexin V staining were produced by DNase I (Roche) or camptothecin (Sigma) treatment, respectively.

\section{Flow cytometric analysis of cell size}

To determine relative size of individual cells, neurosphere cultures were collected in bulk into SFM, dissociated mechanically into single cells, and transferred to PBS. Separate single-cell suspensions were analyzed using forward and side scatter on an EPICS Elite Cell Sorter (Beckman-Coulter). Dead cells were eliminated using dot-plots of forward versus side scatter, and clumps were removed using integrated signal versus peak signal for forward scatter.

\section{Statistical analyses}

Factorial design analysis of variance (ANOVA) or $t$-tests were used to analyze data as appropriate. Significant ANOVA values were followed by simple main effects analyses or post hoc comparisons of individual means using the Tukey method where appropriate. The level of significance for all comparisons was 0.05 .

\section{Acknowledgments}

Thanks to Tania Alexson and other members of our laboratory for comments on earlier versions of this paper, and to Brenda Coles for technical assistance. This work was supported by a Canadian Institute of Health Research grant to D.v.d.K. and a Natural Science and Engineering Research Council fellowship to T.E.K.

\section{References}

Allsopp, R.C., Cheshier, S., and Weissman, I.L. 2001. Telomere shortening accompanies increased cell cycle activity during serial transplantation of hematopoietic stem cells. J. Exp. Med. 193: 917-924.

Brazel, C.Y., Felling, R.J., and Levison, S.W. 2004. A novel method to enrich for neural stem cells. J. Neurochem. 90: $\mathrm{s} 1-\mathrm{s} 47$.
Bringold, F. and Serrano, M. 2000. Tumor suppressors and oncogenes in cellular senescence. Exp. Gerontol. 35: 317-329.

Cheng, T., Rodrigues, N., Shen, H., Yang, Y., Dombkowski, D., Sykes, M., and Scadden, D.T. 2000. Hematopoietic stem cell quiescence maintained by $21^{\text {cip } 1 / \text { waf } 1}$. Science 287: 1804 1808.

Coggeshall, R.E. and Lekan, H.A. 1996. Methods for determining numbers of cells and synapses: A case for more uniform standards or review. J. Comp. Neurol. 364: 6-15.

Doetsch, F., Verdugo, J.M., Caille, I., Alvarez-Buylla, A., Chao, M.V., and Casaccia-Bonnefil, P. 2002. Lack of the cell-cycle inhibitor $\mathrm{p} 27^{\mathrm{Kip} 1}$ results in selective increase of transit-amplifying cells for adult neurogenesis. J. Neurosci. 22: 22552264.

Enwere, E., Shingo, T., Gregg, C., Fujikawa, H., Ohta, S., and Weiss, S. 2004. Aging results in reduced epidermal growth factor receptor signaling, diminished olfactory neurogenesis, and deficits in fine olfactory discrimination. I. Neurosci. 24: 8354-8365.

Ferron, S., Mira, H., Franco, S., Cano-Jaimez, M., Bellmunt, E., Ramirez, C., Farinas, I., and Blasco, M.A. 2004. Telomere shortening and chromosomal instability abrogates proliferation of adult but not embryonic neural stem cells. Development 131: 4059-4070.

Gage, F.H. 2000. Mammalian neural stem cells. Science 287: 1433-1438.

Gardner, R.V., Astle, C.M., and Harrison, D.E. 1997. Hematopoietic precursor cell exhaustion is a cause of proliferative defect in primitive hematopoietic stem cells (PHSC) after chemotherapy. Exp. Hematol. 25: 495-501.

Giardino, L., Bettelli, C., and Calza, L. 2000. In vivo regulation of precursor cells in the subventricular zone of adult rat brain by thyroid hormone and retinoids. Neurosci. Lett. 295: $17-20$.

Groszer, M., Erickson, R., Scripture-Adams, D.D., Lesche, R., Trumpp, A., Zack, J.A., Kornblum, H.I., Liu, X., and Wu, H. 2001. Negative regulation of neural stem/progenitor cell proliferation by the Pten tumor suppressor gene in vivo. Science 294: 2186-2189.

Harley, C.B., Futcher, A.B., and Greider, C.W. 1990. Telomeres shorten during ageing of human fibroblasts. Nature 345: 458-460.

Hayflick, L. 1965. The limited in vitro lifetime of human diploid cell strains. Exp. Cell Res. 37: 614-636.

. 2000. The illusion of cell immortality. Br. J. Cancer 83: 841-846.

Hitoshi, S., Alexson, T., Tropepe, V., Donoviel, D., Elia, A.J., Nye, J.S., Conlon, R.A., Mak, T.W., Bernstein, A., and van der Kooy, D. 2002. Notch pathway molecules are essential for the maintenance, but not the generation, of mammalian neural stem cells. Genes \& Dev. 16: 846-858.

Kippin, T.E., Cain, S.W., Masum, Z., and Ralph, M.R. 2004. Neural stem cells show bidirectional experience-dependent plasticity in the perinatal mammalian brain. J. Neurosci. 24: 2832-2836.

Lois, C. and Alvarez-Buylla, A. 1994. Long-distance neuronal migration in the adult mammalian brain. Science 264: 11451148.

Luskin, M.B. 1993. Restricted proliferation and migration of postnatally generated neurons derived from the forebrain subventricular zone. Neuron 11: 173-189.

Martens, D.J., Tropepe, V., and van der Kooy, D. 2000. Separate proliferation kinetics of fibroblast growth factor-responsive and epidermal growth factor-responsive neural stem cells within the embryonic forebrain germinal zone. J. Neurosci. 20: 1085-1095. 
Martens, D.M., Seaberg, R.S., and van der Kooy, D. 2002. In vivo infusions of exogenous growth factors into the fourth ventricle of the adult mouse brain increase the proliferation of neural progenitors around the fourth ventricle and the central canal of the spinal cord. Eur. J. Neurosci. 16: 1045-1057.

Maslov, A.Y., Barone, T.A., Plunkett, R.J., and Pruitt, S.C. 2004. Neural stem cell detection, characterization, and age-related changes in the subventricular zone of mice. J. Neurosci. 24: $1726-1733$.

Mathon, N.F., Malcolm, D.S., Harrisingh, M.C., Cheng, L., and Lloyd, A.C. 2001. Lack of replicative senescence in normal rodent glia. Science 291: 872-875.

Mauch, P., Constine, L., Greenberger, J., Knospe, W., Sullivan, J., Liesveld, J.L., and Deeg, H.J. 1995. Hematopoietic stem cell compartment: Acute and late effects of radiation therapy and chemotherapy. Int. I. Radiat. Oncol. Biol. Phys. 31: 1319-1339.

Molofsky, A.V., Pardal, R., Iwashita, T., Park, I.K., Clarke, M.F., and Morrison, S.J. 2003. Bmi-1 dependence distinguishes neural stem cell self-renewal from progenitor proliferation. Nature 425: 962-967.

Morshead, C.M. and van der Kooy, D. 1992. Postmitotic death is the fate of constitutively proliferating cells in the subependymal layer of the adult mouse brain. J. Neurosci. 12: 249256.

- 2004. Disguising adult neural stem cells. Curr. Opin. Neurobiol. 14: 125-131.

Morshead, C.M., Reynolds, B.A., Craig, C.G., McBurney, M.W., Staines, W.A., Morassutti, D., Weiss, S., and van der Kooy, D. 1994. Neural stem cells in the adult mammalian forebrain: A relatively quiescent subpopulation of subependymal cells. Neuron 13: 1071-1082.

Morshead, C.M., Craig, C.G., and van der Kooy, D. 1998. In vivo clonal analyses reveal the properties of endogenous neural stem cell proliferation in the adult mammalian forebrain. Development 125: 2251-2261.

Morshead, C.M., Benveniste, P., Iscove, N.N., and van der Kooy, D. 2002. Hematopoietic competence is a rare property of neural stem cells that may depend on genetic and epigenetic alterations. Nat. Med. 8: 268-273.

Morshead, C.M., Garcia, A.D., Sofroniew, M.V., and van Der Kooy, D. 2003. The ablation of glial fibrillary acidic proteinpositive cells from the adult central nervous system results in the loss of forebrain neural stem cells but not retinal stem cells. Eur. J. Neurosci. 18: 76-84.

Musunuru, K. and Hinds, P.W. 1997. Cell cycle regulators in cancer. Karger Landes Systems, Basel, NY.

Ogawa, M. 1994. Differentiation and proliferation of hematopoietic stem cells. Blood 81: 2844-2853.

Oulton, R. and Harrington, L. 2000. Telomeres, telomerase, and cancer: Life on the edge of genomic stability. Curr. Opin. Oncol. 12: 74-81.

Overturf, K., al-Dhalimy, M., Ou, C.N., Finegold, M., and Grompe, M. 1997. Serial transplantation reveals the stemlike regenerative potential of adult mouse hepatocytes. Am. J. Pathol. 151: 1273-1280.

Presnell, S.C., Petersen, B., and Heidaran, M. 2002. Stem cells in adult tissues. Cell Dev. Biol. 13: 369-376.

Qiu, J., Takagi, Y., Harada, J., Rodrigues, N., Moskowitz, M.A., Scadden, D.T., and Cheng, T. 2004. Regenerative response in ischemic brain restricted by $\mathrm{p} 21^{\text {cip1/waf1 }}$. I. Exp. Med. 199: 937-945.

Reynolds, B.A. and Weiss, S. 1996. Clonal population analyses demonstrate that an EGF-responsive mammalian embryonic CNS precursor is a stem cell. Dev. Biol. 175: 1-13.

Seaberg, R.M. and van der Kooy, D. 2002. Adult rodent neuro- genic regions: The ventricular subependyma contains neural stem cells, but the dentate gyrus contains restricted progenitors. J. Neurosci. 22: 1784-1793.

2003. Stem and progenitor cells: The premature desertion of rigorous definitions. Trends Neurosci. 26: 125-131.

Seaberg, R.M., Smukler, S.R., and van der Kooy, D. 2005. Intrinsic differences distinguish transiently neurogenic progenitors from neural stem cells in the early postnatal brain. Dev. Biol. 278: 71-85.

Seoane, J., Le, H.V., and Massague, J. 2002. Myc suppression of the $\mathrm{p} 21^{\text {cip } 1} \mathrm{cdk}$ inhibitor influences the outcome of the p53 response to DNA damage. Nature 419: 729-734.

Serrano, M. 1997. The tumor suppressor protein p16 ${ }^{\mathrm{INK} 4 \mathrm{a}}$. Exp. Cell Res. 237: 7-13.

Shay, J.W. and Wright, W.E. 2000. Hayflick, his limit, and cellular ageing. Nat. Rev. Mol. Cell. Biol. 1: 72-76.

Tang, D.G., Tokumoto, Y.M., Apperly, J.A., Lloyd, A.C., and Raff, M.C. 2001. Lack of replicative senescence in cultured rat oligodendrocyte precursor cells. Science 291: 868-871.

Tropepe, V., Sibilia, M., Ciruna, B.G., Rossant, J., Wagner, E.F., and van der Kooy, D. 1999. Distinct neural stem cells proliferate in response to EGF and FGF in the developing mouse telencephalon. Dev. Biol. 208: 166-188.

van der Kooy, D. and Weiss, S. 2000. Why stem cells? Science 287: 1439-1441.

Vidal, A. and Koff, A. 2000. Cell-cycle inhibitors: Three families united by a common cause. Gene 247: 1-15.

Wagner, M., Hampel, B., Hutter, E., Pfister, G., Krek, W., Zwerschke, W., and Jansen-Durr, P. 2001. Metabolic stabilization of p27 in senescent fibroblasts correlates with reduced expression of the F-box protein Skp2. Exp. Gerentol. 37: 4155.

Weiss, S. and van der Kooy, D. 1998. CNS stem cells: Where's the biology (a.k.a. beef)? J. Neurobiol. 36: 307-314.

Weissman, I.L. 2000. Translating stem and progenitor cell biology to the clinic: Barriers and opportunities. Science 287: $1442-1446$.

. 2001. Stem progenitor cells: Origins, phenotypes, lineage commitments, and transdifferentiation. Annu. Rev. Dev. Biol. 17: 387-403. 


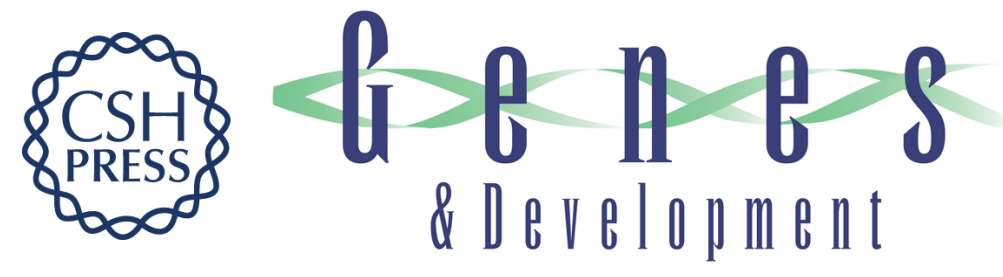

\section{p21 loss compromises the relative quiescence of forebrain stem cell proliferation leading to exhaustion of their proliferation capacity}

Tod E. Kippin, David J. Martens and Derek van der Kooy

Genes Dev. 2005, 19:

Access the most recent version at doi:10.1101/gad.1272305

Supplemental
Material $\quad$ http://genesdev.cshlp.org/content/suppl/2005/03/02/19.6.756.DC1

References This article cites 50 articles, 20 of which can be accessed free at:

http://genesdev.cshlp.org/content/19/6/756.full.html\#ref-list-1

License

Email Alerting Receive free email alerts when new articles cite this article - sign up in the box at the top

Service right corner of the article or click here.

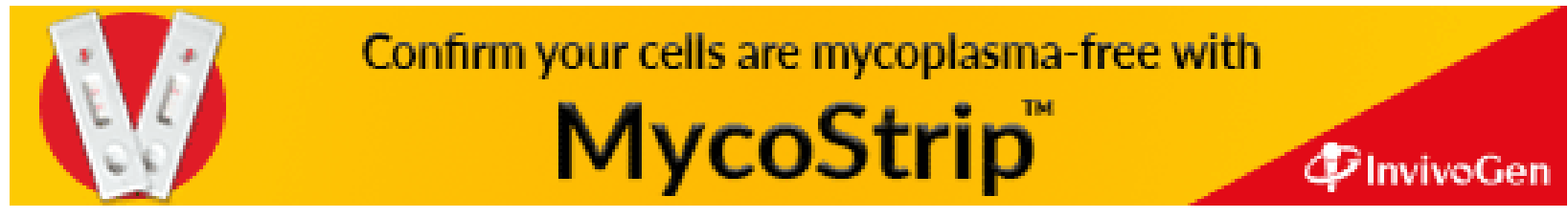

